Editorial

\section{Advanced online publication}

\section{Rossor}

M ost medical journals have significant delays between manuscript acceptance and publication in print due, in part, to unavoidable practical reasons. Publishing articles online ahead of the print version can circumvent this delay and make important clinical and scientific data quickly available. The $B M J$ introduced its advanced online publication section in December 2003 and was followed by the Annals of Rheumatic Diseases as the first of the BMJ specialist journals. The JNNP introduces Online First this month.
The editorial team will select one or two articles per week for advance publication. It will choose those that it considers may have particular impact for clinicians, patients, and researchers. As the process beds in, we hope to increase the number of articles chosen. Authors will be asked for their permission to be part of JNNP Online First and they will have an opportunity to proof the manuscript before publication. The unedited manuscripts will be published on a weekly basis; edited, typeset versions may be posted as they become available. The final print version will be stamped with the JNNP Online First logo and it will be highlighted in the Table of Contents within the issue. The final print version will include the date of initial online publication and all versions will be linked online. All articles will be assigned a unique code-digital object identifier (DOI)and guidance on how to cite the article will appear on the website.

The Online First articles will be indexed by PubMed/Medline within days of publication, thus establishing primacy for the work. The articles will also be searchable through the usual search engines and via JNNP online; the search results will default to the most recent version. Publishing online ahead of print has proved a valuable resource, bringing results rapidly to the public. We are pleased that the JNNP can now offer this service.

J Neurol Neurosurg Psychiatry 2005;76:759

\title{
Beyond the holy grail of motor symptoms: deep brain stimulation for Parkinson's disease
}

\section{Samuel, V Voon}

\section{Optimising non-dopaminergic and dopaminergic related motor responses, as well as cognitive and behavioural responses}

\footnotetext{
$\Lambda$

$\mathrm{t}$ a recent international movement disorders meeting, a timely question was raised: "Is dopaminergic related gambling an indication or a contra-indication for subthalamic nucleus deep brain stimulation?" The question captures not only our curiosity on complex pathophysiology but also the very real uncertainties of our clinical practice.

Parkinson's disease (PD) is a neurodegenerative disorder characterised by motor, cognitive, and behavioural symptoms. Bilateral subthalamic nucleus (STN) deep brain stimulation (DBS) has been available for the treatment for advanced PD since 1993 and has demonstrated marked and sustained efficacy in motor symptoms. ${ }^{1}$ In this issue, the papers by Czernecki et al $(p p$ 775), Colnat-Coulbois et al ( $p p$ 780), and Capecci et al ( $p p$ 769) expand the focus beyond the typical dopaminergic motor symptoms with reports on behavioural changes,
}

balance, and overall functional improvement. These are relevant concepts to clinicians wanting to offer comprehensive advice to their patients and to health care providers who fund such resource intensive therapies.

Czernecki et al address the issue of aggravation of motivational changes following STN DBS by comparing nondemented, non-depressed PD patients 10 months following surgery with a matched non-surgical control group on levodopa using an apathy scale, reward sensitivity, and gambling tasks. As a group, there was no overall significant deterioration in apathy following acute DBS, despite 33\% having significant apathy with DBS off. On the contrary, 9/18 patients of the DBS group had an improvement in apathy. Overall, both levodopa and DBS improved apathy grouped scores to the same extent. The study is limited by the lack of preoperative behavioural baseline measures, brevity of the off-stimulation period, and the lack of caregiver information. The DBS group was withdrawn from a relatively small amount of drug (133 mg of levodopa) in the "off" condition, compared with the levodopa group who were withdrawn from $982 \mathrm{mg}$. Despite these limitations, the study suggests that there is no significant evidence that STN stimulation per se acutely worsened apathy. Further, operated patients whose apathy improved differed from operated patients whose apathy did not improve in having shorter disease duration and less severe parkinsonism. Reward sensitivity and the gambling task measures did not change. These findings suggest that medial frontal and orbitofrontal function, as tapped by these assessments, can remain unaffected by STN DBS.

Colnat-Coulbois et al address the issue of balance control following STN DBS. Normal balance involves the central integration of inputs of proprioceptive, vestibular, and visual function to modulate the magnitude of the motor output (the motor response to the act of falling). In $\mathrm{PD}$, it is hypothesised that the inputs are centrally misrepresented, thus resulting in impairments in the output to (rigid) muscles (impaired postural righting mechanisms). The response of axial symptoms to a preoperative levodopa challenge has been shown to be a good predictor for postoperative outcome of axial symptoms and postural stability. ${ }^{2}$ Colnat-Coulbois et al extend this observation by using prospective measurements of static and dynamic posturography in a cohort of STN DBS patients. The responses from a patient's feet and muscles were recorded 
with the patient standing on a tilting platform while undergoing various experimental procedures designed to perturb balance. The authors showed that selective aspects of both static responses and dynamic adaptation improved following DBS. In particular, the postoperative outcome to the combined effect of DBS and a 30\% reduction in levodopa was occasionally better than that of the preoperative outcome with levodopa alone. The authors hypothesise that the effect of DBS was synergistic with levodopa, possibly mediated via brainstem non-dopaminergic pathways. If indeed the case, it suggests that the current criteria for predicting the outcome of axial symptoms and posture may be too restrictive.

Capecci et al reported a prospective study utilising three different clinicianrated and patient-rated measures of functional status in a cohort of PD patients assessed 1 and 2 years after STN DBS. The results were compared with a matched control group who opted for medical therapy. The authors reported significant improvements in two of the functional scales compared to preoperative baseline measures in the surgical group. In comparison to the control group, the surgical group had significant improvements on all three measures of functional status following surgery due to the decline in function in the control group. The authors argue that in a medically treated group, it may have been unethical to delay by up to 2 years: "treatment whose efficacy in controlling motor symptoms is firmly established". Despite non-blinding, this study highlights not only an outcome clinically relevant to both patients and caregivers, but also the role of control groups in the optimal assessment of postoperative outcomes.

These papers contribute to the ongoing refinement of the practice of STN DBS for advanced PD. Beyond the known improvement of motor signs of $\mathrm{PD}$, the evidence is limited regarding the impact of STN DBS on issues of quality of life, caregiver burden, social outcomes, non-motor symptoms, medication-induced behavioural symptoms, and other non-dopaminergic symptoms. Methodological issues addressed include the need for appropriate control groups and difficulties in achieving blinding and randomisation. Implicit within these, is the need to control for the multiple factors involved in the aetiology of these symptoms (which, as highlighted in these papers, include the non-dopaminergic aetiology of symptoms, STN stimulation, the role of medications, functional neuroanatomy of the STN, or the progression of PD). Currently, despite patient selection based on standard DBS inclusion criteria, ${ }^{3}$ DBS clinicians are sometimes faced post-operatively with patients who have had an excellent motor response, no major change in cognition, and yet feel unsatisfied. There are many unanswered non-motor and motor issues relating to DBS. These three studies allow more focused discussion on the increasingly complex issue of optimising not only the dopaminergic related motor responses, but also nondopaminergic related motor responses, as well as cognitive and behavioural responses.

\section{J Neurol Neurosurg Psychiatry \\ 2005;76:759-760.}

doi: 10.1136/jnnp.2004.061911

\section{Authors' affiliations}

M Samuel, Department of Neurology, King's College Hospital, London, UK

V Voon, National Institutes of Neurological

Disorders and Stroke, National Institute of

Health, Bethesda, Maryland, USA

Correspondence to: $M$ Samuel, Department of Neurology, King's College Hospital, Denmark Hill, London, SE5 9RS, UK; mike.samuel@ kingsch.nhs.uk

\section{REFERENCES}

1 Krack P, Batir A, Van Blercom N, et al. Five-year follow-up of bilateral stimulation of the subthalamic nucleus in advanced Parkinson's disease. N Engl J Med 2003;349:1925-34.

2 Welter ML, Houeto JL, Tezenas du MS, et al. Clinical predictive factors of subthalamic stimulation in Parkinson's disease. Brain 2002; 125:575-83

3 Lang $\mathrm{AE}$, Widner $\mathrm{H}$. Deep brain stimulation for Parkinson's disease: patient selection and evaluation. Mov Disord 2002;17(Suppl 3): S94-101.

Stroke

\section{Stroke services, stroke networks: is there an ideal model?}

\section{T Moulin, M Hommel}

\section{Stroke programmes have a vital role to play in educating the public and primary physicians}

$\mathrm{S}$ ince their first development in the 1970s, the clinical effectiveness of stroke units has been demonstrated in many randomised controlled studies and meta-analyses. The rapid broad-scale implementation of stroke units and structured stroke care in general, has been hindered by many factors of a medical and economic nature. This suggests a missed opportunity for patients in medical terms since almost all stroke patients are hospitalised and it also represents an inappropriate allocation of financial resources.

Different models have been proffered to improve the quality of stroke care and rationalise patient management. The Calgary Stroke Programme ${ }^{1}$ (this issue, $p p$ 863) is a good example of a NorthAmerican stroke centre. It is a centralised model, concentrating stroke medical expertise and material resources in a single acute unit. All patients presenting with suspected stroke are dealt with according to specific management guidelines and flow charts. Another tangible benefit for patients is rapid and direct access to a specialised care system allowing them to "bypass" their local hospital or GP.

While there are clear benefits in the North-American model, should such a centralised model be simply transposed to European countries? It is unlikely that this would work in practice, due to this diverse nature of European health systems.

If the Calgary stroke model could be beneficial for patients and financially efficient for the hospital, it describes the course of individual patients and represents just one link in the "vertical" healthcare network. The global aim of this "vertical" part of the network is to provide emergency care while optimising medical management, reducing patient length-of-stay, as well as the prevention of recurrent stroke to minimise the impact of stroke (the right management, at the right time for the right patient).

A geographically-based structure, providing equal access to stroke expertise for all citizens wherever they live, constitutes the "horizontal" part of the network. These public health care objectives have to be shared by doctors and the appropriate health authority representatives in the framework of a long term medicosocial project. $^{2}$ The two parts of the network mandate state-of-the-art IT supports as well as telemedicine (telestroke) videoconferencing and image transfer 
technology. The networks must also be coordinated by a stroke specialist based in the stroke unit-the cornerstone of the networks. ${ }^{3}$ Perhaps combining European and North-American pieces of the jigsaw could be an effective model, as highlighted by the Project for Integrative Stroke Care (TEMPiS) in Munich, Germany. ${ }^{3}$

What can be agreed upon by both American and European stroke teams is that stroke programmes have a vital role to play in educating the public and primary physicians about risk factors, symptom management, and stroke prevention. There is also a consensus on the need to establish emergency admission procedures, and to organise stroke rehabilitation and long term care to minimise the medico-social impact of stroke.

A firm European endeavour from the healthcare providers and political decision makers will be necessary to achieve this crucial goal.

J Neurol Neurosurg Psychiatry

2005;76:760-761.

doi: 10.1136/jnnp.2004.059121

\section{Authors' affiliations}

T Moulin, Stroke Unit, Dept of Neurology, Jean Minjoz University Hospital, Besançon Cedex, France

M Hommel, Stroke Unit, Dept of Neurology, La Tronche University Hospital, Grenoble Cedex, France
Correspondence to: Dr Thierry Moulin, Stroke Unit, Dept of Neurology, Jean Minjoz University Hospital, 25030 B Besançon Cedex, France; thierry.moulin@univ-fcomte.fr

\section{REFERENCES}

1 Weir N, Buchan AM. Treating stroke and TIA acutely. J Neurology Neurosurg Psychiatry 2005:76:863-5.

2 Hommel M, Deblasi A, Garambois K, et al. The French stroke program. Cerebrovasc Dis 2003;15(Suppl 1):11-3

3 Audebert HJ, Wimmer MU, Schenkel J, et al. Telemedizinisch vernetzte Schlaganfallstationen Nervenarzt 2004:75:161-5.

4 Moulin T, Decavel P, Belahsen F, et al. Information Technology (IT) support in the Implementation of a Stroke Programme: the RUNStroke experiment. Cerebrovasc Dis 2004;17(Suppl 5):6.

\section{Long lasting antalgic effects of daily repetitive transcranial magnetic stimulation in neuropathic pain}

\section{S Owen, T Z Aziz}

\section{Prolonged and significant pain relief can be achieved with repetitive TMS}

S ince the report in 1990 by Tsubokawa $^{1}$ that epidural motor cortex stimulation was effective in alleviating neuropathic pain, there have been multiple reports of the technique with varying results. A predictive test to improve outcome would have been invaluable in selecting patients for such procedures. Transcranial magnetic stimulation (TMS) in single sessions were reported to have a predictive effect by Migita $^{2}$, Canavero ${ }^{3}$, Lefaucheur ${ }^{4}$, and others. However, the effects of such a technique over longer periods of time in a large number of patients have not been reported until now (Khedr et $a l^{5}$; this issue, pp 833).

Using a higher frequency of stimulation $(20 \mathrm{~Hz}$ for five daily sessions) Khedr et al were able to achieve an average $45 \%$ pain reduction compared with sham stimulation (5\%), as assessed by visual analogue score (VAS) and Leeds assessment of neuropathic symptoms and signs (LANSS) questionnaires. The reduction is significantly higher than that reported by Lefaucheur ${ }^{4}$ using $10 \mathrm{~Hz}$ stimulation. The number of patients studied was large-24 had trigeminal neuralgia, and 24 had post stroke pain. Significant pain relief occurred up to 2 weeks after the last session and the degree of pain relief increased with the number of sessions.

This paper is significant for a number of reasons. At first glance it only confirms what has already been reported. However, it goes further. Prolonged and significant pain relief can be achieved with repetitive TMS. In this series the majority had head, face, and arm pain, either from trigeminal neuralgia or stroke. There were a few non-responders and I wonder whether this is related to stroke as the authors point out. Certainly this has been our experience. It may also be more difficult to achieve pain relief in the leg region or to cover hemi body pain that occurs after stroke due to inaccessibility of the leg area to TMS. It is also of interest that although stimulation was applied over the hand and arm area there was a good response in patients with facial pain. It is also of interest that although motor cortex stimulation generally is applied at about $40 \mathrm{~Hz}$ across groups, TMS uses much lower frequencies-20 Hz in this study.

This may well establish higher frequency TMS as an effective methodology of screening patients for implantation of motor cortex stimulation and since the effects are rather prolonged, may even prove to be a methodology that may be useful as an outpatient treatment for certain pain patients.

Perhaps, given the effectiveness of this procedure, the afflicted patients have some recourse to continued TMS in Egypt. This would also answer the question of whether this therapy could be offered to many patients without recourse to surgery.

J Neurol Neurosurg Psychiatry 2005;76:761. doi: 10.1136/jnnp.2004.060483

\section{Authors' affiliations}

S L F Owen, University Department of Physiology, University of Oxford, Oxford, UK T Z Aziz, Nuffield Department of Surgery, University of Oxford, Oxford, UK

Correspondence to: Professor T Z Aziz, Department of Neurosurgery, Radcliffe Infirmary, Woodstock Road, Oxford OX2 6HE, UK; tipu.aziz@physiol.ox.ac.uk

\section{REFERENCES}

1 Tsubokawa T, Katayama Y, Yamamoto T, et al. Chronic motor cortex stimulation for the treatment of central pain. Acta Neurochir Suppl (Wien) 1991;52:137-9.

2 Migita K, Uozumi T, Arita K, et al. Transcranial magnetic coil stimulation of motor cortex in patients with central pain. Neurosurgery 1995;36:1037-9, discussion 1039-40.

Canavero S, Bonicalzi V, Dotta $M$, et al. Transcranial magnetic cortical stimulation relieves central pain. Stereotact Funct Neurosurg 2002;78:192-6.

4 Lefaucheur J-P, Drouot X, Menard-Lefaucheur I, et al. Neurogenic pain relief by repetitive transcranial magnetic cortical stimulation depends on the origin and the site of pain. I Neurol Neurosurg Psychiatry 2004;75:612-6.

5 Khedr EM, Kotb H, Nageh K, et al. Long lasting antalgic effects of daily sessions of repetitive transcranial magnetic stimulation in central and peripheral neuropathic pain. J Neurol Neurosurg Psychiatry 2005;76:833-8 